\title{
ERRATUM
}

\section{The origin and evolution of maize in the Southwestern United States}

Rute R. da Fonseca, Bruce D. Smith, Nathan Wales, Enrico Cappellini, Pontus Skoglund, Matteo Fumagalli, José Alfredo Samaniego, Christian Carøe, María C. Ávila-Arcos, David E. Hufnagel, Thorfinn Sand Korneliussen, Filipe Garrett Vieira, Mattias Jakobsson, Bernardo Arriaza, Eske Willerslev, Rasmus Nielsen, Matthew B. Hufford, Anders Albrechtsen, Jeffrey Ross-Ibarra and M. Thomas P. Gilbert

Nature Plants 1, 14003 (2015); published 8 January 2015; corrected 14 January 2015.

In the version of the Supplementary Information originally posted online, the Methods section was missing. This has been corrected 14 January 2015. 